\title{
Turbulent Mixing in Wind-Blown HII Regions
}

\author{
D. Breitschwerdt ${ }^{\dagger}$ and F.D. Kahn ${ }^{\ddagger}$ \\ ${ }^{\dagger}$ Max-Planck-Institut für Kernphysik, D-6900 Heidelberg, Postfach 103980, FRG \\ ${ }^{\ddagger}$ Department of Astronomy, The University, Manchester M19 9PL,England
}

\begin{abstract}
Turbulent mixing between an ionization bounded HII region and a hot shocked stellar wind (HSSW), which keeps it under pressure, is examined. Recently we have shown that acoustic disturbances can grow there to finite amplitude in a time scale which is comparable to the sound crossing time in the HII layer. The resulting turbulence will then stretch fluid elements and the frozen-in magnetic field. A condition under which turbulence can decay down to the viscous scale, where mixing is very efficient, is derived. For a uniform and plane parallel magnetic field $B_{0}$ and a constant density $\rho_{0}$ of the ambient medium, we find that efficient mixing takes place near the polar regions. Subsequently the rate of mass addition to the hot bubble is calculated and it is shown that catastrophic cooling is likely to occur. In the case of NGC 6334(A) it seems that this has just happened and we predict an upper limit for $B_{0}$ of $4 \times 10^{-5}$ gauss there. This model may also explain the existence of highly ionized species (e.g. OVI), soft $\mathrm{X}$-rays and high velocity flows of the order of $100 \mathrm{~km} / \mathrm{s}$.
\end{abstract}

\section{Introduction}

According to well-known theoretical models (e.g. Dyson and deVries, 1972; Castor et al., 1975; Dyson, 1989) OB stars and Planetary Nebulae should generally have HII regions which are squeezed into thin shells held under pressure by hot shocked stellar winds (HSSWs). However, quite often observations show rather extended HII regions, such as the Rosette Nebula (Dickel 1974) or NGC 6334(A) (Rodriguez et al. 1988). In addition, the classical model fails to explain high velocity flows in excess of $100 \mathrm{~km} / \mathrm{s}$ (López et al. 1989), the existence of highly ionized species, such as OVI, NV and also soft X-rays. These are indicative of temperatures of the order of $10^{5}-10^{6} \mathrm{~K}$, which are neither present in the hot bubble $\left(>10^{7} \mathrm{~K}\right)$ nor in the HII region $\left(\approx 10^{4} \mathrm{~K}\right)$.

In a recent paper (Breitschwerdt and Kahn, 1988) we have shown that acoustic disturbances travelling parallel to the HII/HSSW interface can grow to finite amplitude provided that the radiation is not exactly incident at right angles and that the HII region is ionization bounded. Hence the classical model is unstable and the growth rate of waves is fairly large, viz. inversely proportional to the sound crossing time in the HII layer. We will show in the following that the resulting turbulence can induce local mixing and cooling, thus altering the dynamics of wind-blown HII regions drastically. 


\section{Model for Turbulent Mixing with Magnetic Field}

We assume that the wind expands into an ambient medium with a magnetic field which is uniform and plane parallel on a scale of tens of parsecs. It should also be sufficiently weak, so that similarity solutions for the hot bubble are still applicable.

At largest scale $l$, which corresponds to the thickness of the HII layer, the magnetic energy density shall be less than the turbulent energy density. All the speeds $u$ are less than the isothermal speed of sound $c_{i}$ in the ionized gas, so that the turbulent flow is incompressible and the characteristic speed at length scale $l^{\prime}$ follows a Kolmogorov law

$$
u^{\prime}=u\left(\frac{l^{\prime}}{l}\right)^{1 / 3}
$$

The effect of turbulence is to stretch fluid elements and the frozen-in magnetic field which, viewed on smaller scales, leads to an increase in surface area and field strength. However, there will also be a reduction of magnetic energy density due to magnetic reconnection.

Let us divide the length scales into octaves:

$$
\frac{l}{l^{\prime}}=2^{n}
$$

and let $B_{n}^{2}$ be the mean squared field at the $n^{\text {th }}$ octave. It is then easy to see that in the absence of reconnection $B_{n}^{2}$ scales like

$$
B_{n}^{2}=B_{i}^{2}\left(\frac{l}{l^{\prime}}\right)^{1 / 3}=B_{i}^{2} e^{\eta n},
$$

where $\eta=(1 / 3) \log 2$.

Therefore the change in $B_{n}^{2}$ from octave $n$ to $n+1$ in the presence of magnetic reconnection is

$$
\frac{d B_{n}^{2}}{d n}=\eta B_{n}^{2}-C\left(\frac{d B_{n}^{2}}{d n}\right) \frac{B_{n}}{l \cdot \sqrt{4 \pi \rho_{i}}} \frac{l}{u} .
$$

$\rho_{i}$ is the mass density in the HII region and $C$ is a proportionality constant usually taken to be 0.1 (Petschek 1964). In this paper we prefer the value 0.05 since the lines of force are not necessarily antiparallel here.

For convenience we write $B_{n}=f B_{i}$ and $\sqrt{4 \pi \rho_{i} u^{2}} / B_{i}=M_{A}$, the Alfvén Mach number. In a forthcoming paper (Kahn and Breitschwerdt, 1989) we solve equation (4) in terms of $f$. Here we just briefly discuss the two asymptotic limits for small and for large $n$.

In the first case the solution without reconnection is recovered

$$
f=e^{\eta n / 2}
$$

and stretching is the dominant process. For large $n$, i.e. small length scales, reconnection is more important and we find

$$
f=\left(\frac{7 M_{A}}{C}\right)^{8 / 7} e^{-5 \eta n / 2} .
$$

This leads to the conclusion that there is a critical length scale $l_{c}^{\prime}$, where the magnetic energy density has a maximum, which is given by

$$
\frac{l}{l_{c}^{\prime}} \equiv\left(\frac{7 M_{A}}{C}\right)^{6 / 7} \text {. }
$$


Thus on smaller scales the magnetic field strength becomes progressively weaker and turbulence can cascade down until viscous dissipation takes place.

In order that this process works, two important restricions have to be imposed. First, the accumulated rate of dissipated magnetic energy down to scale $l_{c}^{\prime}$ due to reconnection must be less than the rate of dissipated mechanical energy. It is shown elsewhere that this can be expressed as $M_{A} \gg 1.8 C^{-1 / 6} \approx 3$. Second, the magnetic energy density must always be less than the kinetic energy density on any scale. This criterion yields $M_{A} \gg(7 / C)^{3 / 4} \approx 41$, which is the more compelling.

This will limit the total surface area of the HII/HSSW interface, where efficient mixing can take place, simply because the magnetic field has to be weak enough to satisfy the above conditions.

\section{Mixing Rate and Catastrophic Cooling}

Using our assumptions of the preceding section we describe the large scale expansion of the bubble by similarity solutions (e.g. Dyson 1981):

$$
R_{b}=0.76\left(\frac{L_{w}}{\rho_{0}}\right)^{1 / 5} t^{3 / 5}
$$

where $R_{b}$ is the radius of the bubble and $L_{w}$ is the wind mechanical luminosity and

$$
\rho_{i}=0.78 \rho_{0}\left(\frac{\dot{R}_{b}}{c_{i}}\right)^{2}
$$

Here and in the following all dimensional quantities are in CGS units. For a plane parallel magnetic field geometry, the field strength in the compressed HII region, $B_{i}$, varies with polar angle like

$$
B_{i}=1.15 B_{0}\left(\frac{\dot{R}_{b}^{2}}{c_{i}^{2}}\right) \sin \theta .
$$

This follows from conservation of magnetic flux across the shell and the thicknes $l$ of the ionized part (which has been used in equation (9)) can be derived from ionization balance.

A typical growth rate for unstable waves in the ionized layer is $\sigma=0.1 c_{i} / l$ and therefore the rate at which energy is fed into the largest eddies is $0.1 c_{i} u^{2} / l$, which when equated to the rate of dissipation, yields $u=0.13 c_{i}$. This value has to be used when the above condition $M_{A}>41$ is applied. It implies that there is a critical field strength $B_{i}$ or by virtue of equation (9) a critical angle $\theta_{c}$, increasing with time, so that for $\theta<\theta_{c}$ free mixing occurs at a rate $0.13 \rho_{i} c_{i}$ per unit area. In our model with its simple field configuration this happens near the polar caps of the HII/HSSW interface and the rate of mass addition to the bubble is

$$
\dot{\mathcal{M}}=2.7 \times 10^{-5} L_{w}^{2 / 5} \rho_{0}^{8 / 5} c_{i}^{3} B_{0}^{-2} t^{6 / 5} .
$$

The flow of cooler $\left(10^{4} \mathrm{~K}\right)$ gas per unit time overwhelms the HSSW gas generated by the terminal stellar wind shock by a large factor and therefore induces local radiative cooling in an otherwise always adiabatic bubble. A catastrophic loss of pressure near the polar caps seems unavoidable and therefore large scale motions should occur. It is interesting to note that the magnetic field geometry automatically produces bipolar outflow structures once the shell breaks up; these have been observed in many cases. 
If the mass of the bubble is obtained in this fashion, an averaged density and pressure and thus the time scale for radiative cooling, $t_{c}$, can be worked out. Cooling of the bubble may become catastrophic in the sense that its expansion is severly disturbed and it may lead to a collapse in the extreme case. This happens when $t \leq t_{c}$ or

$$
t \geq t_{c r i t} \equiv 2.73 \times 10^{9}\left(\frac{\rho_{0}}{\rho_{-24}}\right)^{-23 / 16}\left(\frac{L_{w}}{L_{36}}\right)^{11 / 32}\left(\frac{B_{0}}{B_{-6}}\right)^{25 / 18} \mathrm{yr}
$$

where $c_{i}=10 \mathrm{~km} / \mathrm{s}$ has been used and $\rho_{-24}, L_{36}$ and $B_{-6}$ are $10^{-24} \mathrm{~g} / \mathrm{cm}^{3}, 10^{36} \mathrm{erg} / \mathrm{s}$ and $10^{-6}$ gauss, respectively.

To illustrate this effect, we apply these results to NGC 6334(A), which has been observed by Rodriguez et al. (1988). They infer $L_{w}=10^{36} \mathrm{erg} / \mathrm{s}, \rho_{0}=4 \times 10^{-19} \mathrm{~g} / \mathrm{cm}^{3}$, $R_{b}=3.7 \times 10^{17} \mathrm{~cm}, l=0.1 R_{b}$ and a Ly c photon rate $S_{*}=4 \times 10^{48} \mathrm{~s}^{-1}$, which yields an age of about $2.2 \times 10^{11} \mathrm{~s}$. It can be shown easily that the HII region is ionization bounded and therefore our model holds. The bubble on the radio map appears slightly distorted and is embedded in a bipolar flow. Therefore our tentative conclusion is that catastrophic cooling has just occured but it has not yet destroyed the structure. Alternatively, the loss of pressure increases the thickness of the HII layer and therefore the growth time of the instability as well. As a consequence the mixing rate drops and therefore cooling becomes less efficient. In any case it allows us to set an upper limit to the magnetic field strength in the surrounding molecular cloud:

$$
B_{0} \leq 4 \times 10^{-5} \text { gauss. }
$$

It would be highly desirable to obtain measurements both of the field strength and its orientation for NGC 6334 (A) or the ambient media of other early type stars.

\section{Conclusions}

We suggest that turbulent mixing around early type stars should be a common phenomenon. In the view of the obstruction of conductive heat flux by even weak magnetic fields it is a possible way of exchanging mass and energy between the hot bubble and the HII region. It has been shown that the mixing rate itself depends mainly on $B_{0}$ and $\rho_{0}$ and only weakly on stellar parameters such as the Ly c photon rate or the mechanical luminosity of the wind. The model presented here can explain naturally the existence of OVI and NV lines and soft X-rays which should arise in cooling regions and also high velocity flows, which we are currently investigating.

\section{References}

Breitschwerdt, D. and Kahn, F.D., 1988, Mon. Not. R. astr. Soc., 235, 1011.

Castor, J., McCray, R., and Weaver, R., 1975, Astrophys. J., 200, L107.

Dickel, R.H., 1974, Astr. Astrophys., 31, 11.

Dyson, J.E., 1981, Investigating the Universe, ed. Kahn, F.D., D. Reidel Publ. Comp., Dordrecht, Holland, p.125.

Dyson, J.E., 1989, these proceedings.

Dyson, J.E. and deVries, J., 1972, Astr. Astrophys., 20, 223.

Kahn, F.D. and Breitschwerdt, D., 1989, Mon. Not. R. astr. Soc., (submitted).

López, J.A., Falcón, L.H., Ruiz, M.T. and Roth, M., 1987, Planetary Nebulae, IAU

Symposium No. 131, eds. Torres-Peimbert, S., Kluwer Acad. Publ. Comp., Dordrecht, p.179.

Petschek, H.E., 1964, NASA SP-50, U.S. Govt. Printing Office, p.425.

Rodriguez, L.F., Cantó, J. and Moran, J.M., 1988, Astrophys. J., 333, 801. 


\section{Discussion:}

DYSON: Do your results imply that energy driven bubbles are rather rare?

BREITSCWERDT: At present I cannot give a clearcut answer. The mixing process which I have discussed drives the bubble into the momentum driven phase for some time. However the following increase in thickness of the HII region reduces the instability rate and hence turbulence. At the same time hot gas is fed into the bubble continuosly by the stellar wind and thus raise the pressure there and possibly lead to an energy driven flow again. We are going to investigate whether there exists a self-limiting process or whether the bubble will undergo an oscilatory behaviour switching from momentum to energy driven flow.

JONCAS: In an HII region undergoing a Champagne phase, would the flow of ionized gas eliminate any effect the stellar wind bubble would have? Is it time dependent?

BREITSCWERDT: Certainly not. On the contrary, if there is a density jump at some interface like the edge of a molecular cloud, the formation of a secondary bubble will occur which will also be driven by the stellar wind.

ROY: Could heat conduction play any role in the energy transfer? Why?

BREITSCWERDT: Although heat conduction has been advocated quite often in the past as a mechanism for energy transfer I would like to stress that there are some severe problems. First, any magnetic field with a component perpendicular to the temperature gradient will reduce the mean free path of electrons considerabily. Second, the normal Spitzer conductivity is certainly not apropiate in the case of a very steep temperature gradient where the scale height is less than the mean free path, like at the HII/ bubble interface. 